\title{
Os desafios da estratégia da atenção primária no Amazonas e propostas para melhoria da assistência em saúde: uma revisão integrativa da literatura
}

\author{
The challenges of primary health care in Amazonas and proposals for improving health care: an \\ integrative literature review \\ Los desafíos de la atención primaria de salud en Amazonia y las propuestas para mejorar la \\ atención sanitaria: una revisión integrativa de la literatura
}

\section{Resumo}

Introdução: A atenção básica é definida como um conjunto de ações de saúde que abrangem ações individuais, familiares e coletivas envolvendo promoção, proteção e recuperação da saúde, transformando-se em Programa de Saúde da Família ou Estratégia Saúde da Família, buscando um modelo efetivo da assistência em saúde. Objetivo: apresentar os desafios enfrentados pelo programa de estratégia de saúde da família. Metodologia: Este estudo trata-se de uma revisão integrada de literatura, realizado no período de 10 de janeiro de 2021 à 21 de fevereiro de 2021, nas bases de dados: Lilacs, Pubmed, Scielo, Medline. Resultados e discussão: Identificou-se nos artigos analisados a diversidade de tarefas e ações das equipes ou trabalhadores da estratégia de Saúde da Família, além de desafios encontrados, demonstrados pelos fatores vivenciados pelos profissionais frente a realidade dos municípios do estado do Amazonas. Considerações Finais: Partindo das características geográficas e socioeconômicas voltados para as peculiaridades e modo de vida da população, a atenção primária no Amazonas enfrenta diversidades referente a um cenário limitado de recursos. Portanto, tais fatores exigem maiores investimentos e capacitações aos profissionais além de realização de pesquisas voltadas para a temática, afim de esclarecer impasses encontrados e gerar conhecimento sobre o tema.

Palavras-chave: Estratégia saúde da família; Atenção Primária à Saúde; Amazonas.

\begin{abstract}
Introduction: Primary care is defined as a set of health actions that encompass individual, family and collective actions involving health promotion, protection and recovery, becoming a Family Health Program or Family Health Strategy, seeking and effective care model in health. Objective: to present the challenges faced by the Family health strategy program. Methodology: This study is an integrated literature review, carried out from January 10, 2021 to February 21,2021, in the following databases: Lilacs, Pubmed, Scielo, Medline. Results and discussion: It was identified in the analyzed articles the diversity of tasks and action of the teams or workers of the Family Health strategy, in addition to the challenges encountered, demonstrated by the factors experienced by professionals in the reality of municipalities in the state of Amazonas. Final Considerations: Departing from the geographic and socioeconomic characteristics focused on the peculiarities and way of life of the population, primary care in Amazonas faces diversities regarding a limited resource scenario. Therefore, such factors require greater investment and training for professionals, in addition to conducting research focused on the subject, in order to clarify the impasses found and generate knowledge on the subject.
\end{abstract}

Keywords: Strategy family health; Primary Health Care; Amazonas.

\section{Resumen}

Introducción: La atención primaria se define como un conjunto de acciones de salud que engloban acciones individuales, familiares y colectivas que involucran la promoción, protección y recuperación de la salud, convirtiéndose en un Programa de Salud de la Familia o Estrategia de Salud de la Familia, buscando un modelo eficaz de atención en salud. Objetivo: presentar los desafíos que enfrenta el programa de estrategia de salud de la familia. Metodología: Ese estudio es una revisión integral de la literatura, realizada del 10 de enero de 2021 al 21 de febrero 
de 2021, en las siguientes bases de dados: Lilacs, Pubmed, Scielo, Medline. Resultados y discusión: Se identificó en los artículos analizados la diversidad de tareas y acciones de los equipos o trabajadores de la estrategia Salud de la Familia, además de los desafíos encontrados, demostrados por los factores vividos por los profesionales en la realidad de los municipios del estado de Amazonas. Consideraciones finales: Partiendo de las características geográficas y socioeconómicas enfocadas en las peculiaridades y forma de vida de la población, la atención primaria en Amazonas enfrenta diversidades en un escenario de recursos limitados. Por tanto, dichos factores requieren de una mayor inversión y formación de los profesionales, además de realizar investigaciones centradas en el tema, con el fin de esclarecer los impasses encontrados y generar conocimiento sobre el tema.

Palabras clave: Estrategia salud de la familia; Atención Primaria de Salud; Amazonas.

\section{Introdução}

Atenção Básica no Brasil (AB) é definida como um conjunto de ações de saúde individuais, familiares e coletivas que envolvem promoção, prevenção, proteção, diagnóstico, tratamento, reabilitação, redução de danos, cuidados paliativos e vigilância em saúde (Brasil, 2017). Nos últimos anos tem se transformado a partir do Programa de Saúde da Família (PSF) ou Estratégia Saúde da Família (ESF) reestruturando suas práticas buscando um modelo efetivo da assistência em saúde. Os investimentos para a equipe da Estratégia Saúde da Família trazem a necessidade de reflexão sobre sua concepção, operacionalização e sustentabilidade (Oliveira et al.,2011).

O Amazonas é o maior estado do país, com 1.577.820,2 km2 e 1,79 habitantes/km2, geograficamente apresenta características desfavoráveis que dificultam o acesso aos serviços de saúde. Apresenta problemas de saneamento básico e desigualdades econômicas, além de uma população com características peculiares residentes em áreas urbanas e rurais, sendo ribeirinhos ou indígenas (IBGE, 2009). O clima tropical úmido somando-se às condições acima descritas, deixam a população vulnerável a diversos problemas de saúde como as doenças predominantes destas condições climáticas (Oliveira et al., 2011).

A Atenção Básica em contexto amazônico mostra que a promoção em saúde apresenta inconsistências e limitações para a oferta de um cuidado integral. Ou seja, há necessidade em sustentar estratégias de qualificação dos processos de trabalho, assim como de monitorar os problemas, o que dificulta o cuidado integral em saúde. Para tal, é preciso reconhecer o que tem gerado obstáculos organizacionais e o que poderia promover condições para as equipes de Atenção Primária à Saúde em atuar na perspectiva de um cuidado contínuo e integral. Desta forma, concentrar estratégias para a melhoria das condições (Rabelo et al., 2020).

Municípios distantes da capital do Amazonas possuem uma dificuldade maior de manter a qualidade da Atenção Primária a Saúde, por insuficiência de resolubilidade, por baixa quantidade de profissionais e rotatividade dos mesmos, devido ao difícil acesso e precariedade nos serviços disponíveis. Estes locais são figurados como locais negligenciados e desfavoráveis para exercer tais ações (Dolzane \& Schweickardt, 2020). Reforça-se, assim, a ideia de que o Programa Estratégia de Saúde da Família não está positivamente a favor da população rural, visto que ainda não foram vencidas as barreiras limitantes à completa implantação do programa no estado em que é fortemente latente a questão de territorialização (Oliveira et al., 2011).

Deste modo, este trabalho tem como objetivo apresentar através de uma busca integrativa da literatura os desafios enfrentados pelo programa de estratégia de saúde da família no Amazonas além de apresentar propostas que visem a melhoria da assistência em saúde da população amazonense.

\section{Referencial Teórico}

A AB em saúde por sua atuação com base territorial, ampliação da infraestrutura, pelas equipes multiprofissionais e continuidade das ações tem sido um dos fatores que propiciam a inclusão. A ampliação de equipes de ESF é um fator fundamental para a cobertura dos serviços nos diferentes territórios do país, representando a mais importante política de acesso à saúde na história do Brasil (Silva et al., 2017). Com pouco mais de duas décadas de implantação, a ESF consolidou-se como 
o melhor formato de organização de equipes profissionais e de reorientação das práticas assistenciais na Atenção Primária Saúde no Brasil (Macinko et al., 2015).

A ESF se define por um conjunto de ações e serviços baseadas nas necessidades da população que se estendem além da assistência médica, considerando o estabelecimento de vínculos entre os usuários dos serviços e os profissionais de saúde em contato permanente com o ambiente. Tais princípios e diretrizes fundamentam a Política Nacional de Atenção Básica (PNAB) (Brasil, 2011).

A garantia da qualidade da atenção primária à saúde apresenta-se atualmente como um dos desafios ao Sistema Único da Saúde (SUS) considerando a necessidade desta ser compreendida à luz dos princípios da integralidade, universalidade, equidade e participação social (Brasil, 2011). A e apesar do crescimento significativo da cobertura pela ESF está diretamente implicado no aumento do acesso aos cuidados em saúde e da melhoria dos principais indicadores de saúde da população brasileira; ainda há muitas regiões que enfrentam desafios importantes, considerando a superação das condições desiguais de acesso aos serviços de Saúde (Tonhá et al., 2015) particularmente referidos a equidade do acesso, resolutividade e qualidade da atenção (Fausto, 2013).

A Estratégia de Saúde da Família surgiu com o propósito de ampliar o acesso da população aos serviços básicos de saúde, como uma possibilidade de mudança do modelo assistencial vigente. Todavia, por estar no contexto do SUS, torna-se um grande desafio a ESF não somente ampliar a sua cobertura, mas também dar conta daquilo que já é proposto na atenção primária à saúde. O indicador de cobertura populacional do Estado do Amazonas ainda não tem implantação total de municípios com as ações da ESF (Oliveira et al., 2011).

A AB, no contexto da Amazônia, é dificultada por diversos fatores geográficos e sociais. A extensa rede hidrográfica e dispersão populacional são exemplos de fatores que afetam a programação de atividades e serviços a serem realizados em certas áreas, a não realização pelas limitações encontradas devido às peculiaridades da região, favorecem o elevado número de pessoas ainda desassistidas pelos serviços básicos, o que ocasiona outros agravos nas diversas faixas etárias, especialmente naquelas populações em situação de vulnerabilidade social localizadas nas regiões mais afastadas dos centros urbanos (Guimarães, 2016).

\section{Metodologia}

\subsection{Estratégia de pesquisa e seleção dos estudos}

Este estudo trata-se de uma revisão integrada de literatura do tipo do tipo exploratório, de caráter qualitativo de acordo com Estrela, C. (2018). Realizada no período de 10 de janeiro de 2021 até 22 de fevereiro de 2021 e foram utilizadas as palavras chaves: Estratégia; saúde da família e Amazonas na língua portuguesa e na língua inglesa "amazonas and family and health strategy", nas bases de dados Lilacs, PubMed, SciELO, Medline.

A identificação dos estudos foi de acordo com títulos e resumos envolvendo as palavras chaves. Notou-se que muitos estudos não atendiam nosso principal critério de inclusão, que seria grupos de estratégia da família que realizam trabalhos em qualquer município do Amazonas. Após aplicação dos critérios de elegibilidade, obteve-se uma amostra final de 10 artigos, os quais foram lidos e analisados na íntegra (Figura 1). 
Figura 1: Fluxograma da estratégia de seleção de estudos. Manaus - AM, Brasil, 2021.

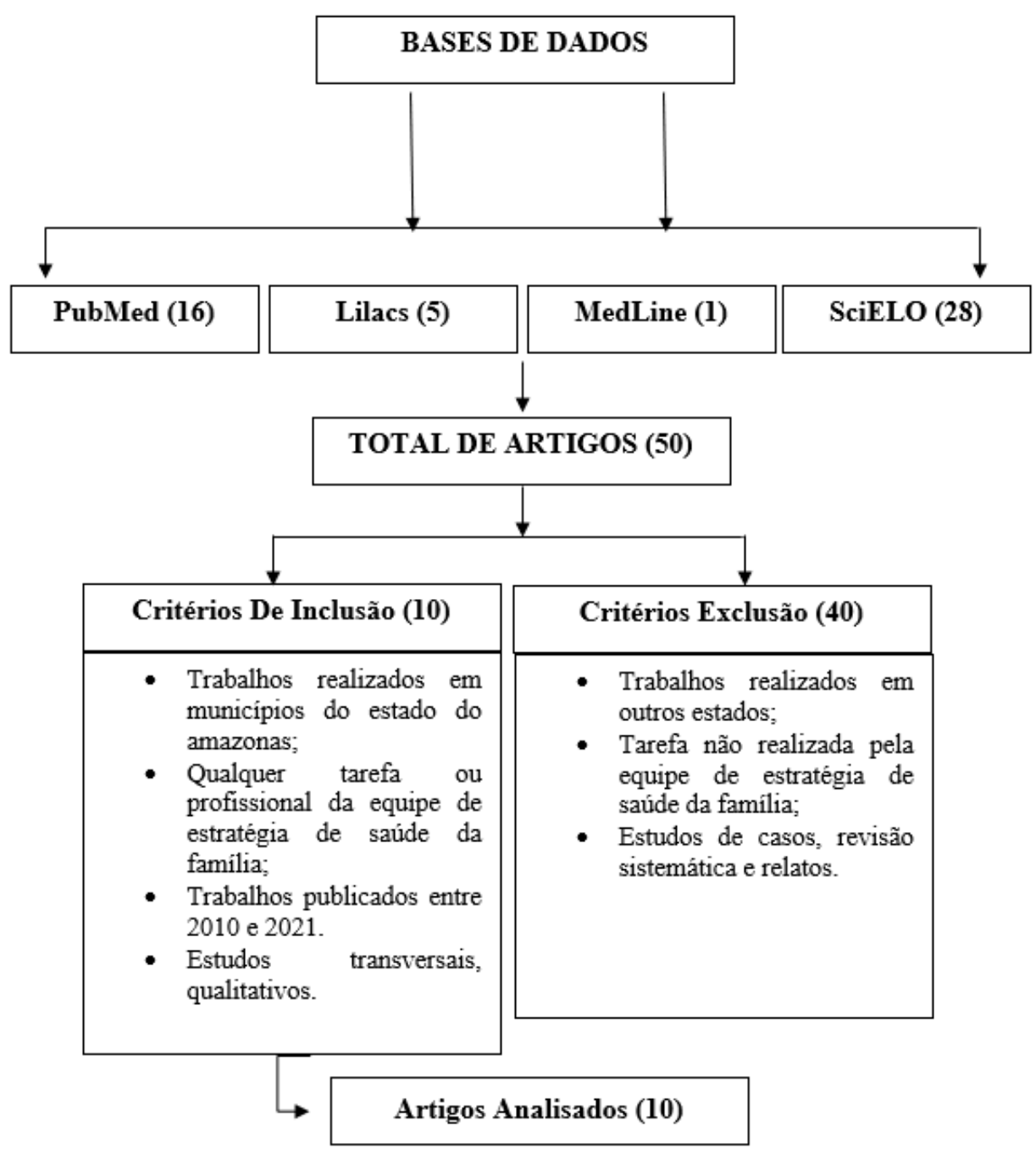

Fonte: Autores (2021).

\section{Resultado e Discussão}

Os 10 artigos analisados neste estudo tratam-se de estudos transversais descritivos e qualitativos, explanando a diversidade de tarefas e ações das equipes ou trabalhadores da estratégia de saúde da família, através de análises, avaliações e entrevistas, demonstrando fatores vivenciados pelos profissionais na realidade dos municípios do estado do Amazonas e os desafios enfrentados.

Durante os anos de 2004 a 2008 o autor caracterizou a implantação da ESF no estado do amazonas por meio de indicadores representativos de cobertura populacional, ações desenvolvidas por equipes e mudanças do programa através de comparação dos dados de doenças mais comuns enfrentadas pela AB. O estudo na época caracterizou como insatisfatória a ESF em todo o estado, demonstrando fragilidade do programa. Este tipo de estudo demonstra grande importância para avaliação e tomadas de decisões a fim de buscar melhorias para o programa de ESF, a escassez de trabalhos que estudem a ESF no Amazonas demonstra que apesar dos avanços, estes ainda enfrentam desafios significativos desde sua implantação. (Oliveira et al., 2011) 
A reestruturação do modelo de assistência da ESF avançou em alguns aspectos, especialmente no que diz a organização política e institucional das equipes, porém ainda se depara com dificuldades relacionadas à alternância e condições precárias de vínculos trabalhistas, além da insuficiente qualificação dos profissionais e necessidades de infraestrutura e materiais, oriundos desde décadas anteriores. Tais limitações implicam na demanda de ações e assistência por exemplo da população com doenças crônicas: hipertensão, diabetes, hanseníase, tuberculose, entre outras que demandam acompanhamento e que no Amazonas demonstrasse precário comparado a outras regiões do Brasil (Garnelo et al., 2014).

O valor do planejamento e programação em saúde é essencial e estimado, mas o trabalho das equipes permanece refém da demanda espontânea e do atendimento clínico curativo individual (Garnelo et al., 2014). Os estudos de Moretti-Pires \& Campos (2010) e Oliveira et al., (2011) realizados distintamente em dois municípios do Amazonas, respectivamente Coari e Manacapuru compreendem que há ausência do trabalho em equipe de fato, representado em um contexto que cada profissional realiza seu trabalho de forma isolada, não associada, o que contribui para conflitos de poderes ou concentração de poder em uma gestão o que detém o trabalho coletivo. A intervenção conjunta da equipe multiprofissional é essencial para caracterização da ESF como proposta diferencial dos modelos antecessores.

As peculiaridades e características geográficas, socioeconômicas do Amazonas já supracitadas neste estudo, são fatores que interferem diretamente na logística, na qualidade, capacitação, nas ações, equipes e no profissional de saúde ou assistência em saúde. As tradicionais populações ribeirinhas da região amazônica, caracterizadas por residirem as margens dos rios em casas planejadas, infraestrutura escassa e com modo de vida adaptado aos fenômenos de inundações e secas, são assistidas pela ESF fluvial, uma adaptação do projeto em que a equipe multiprofissional em algum tipo de embarcação acompanha essa população (Figueira, 2020).

Figueira (2020) demonstra em seu estudo realizado em duas embarcações de equipe de ESF fluvial em região de várzea do amazonas que o trabalho da equipe multiprofissional acontece de forma coletiva e em associação com as unidades familiar da comunidade. Diferente como já observado das regiões urbanas que demonstram ainda dificuldades desta coletividade entre profissionais e entre a população. Ademais salienta a importância da ESF fluvial nessas comunidades isoladas e como a falta de investimentos, materiais, e capacitação implica diretamente na qualidade e maior dificuldade da realização de manutenção do trabalho e acesso. O custeio mensal de uma UBS fluvial gira em média 80 mil reais ao mês. Em um município do Amazonas de médio porte conta com o quantitativo de 25 equipes de Atenção primária na zona urbana, o custo varia em torno de 398.059.36 reais por mês (e-gestor, 2020).

Tais fatores também são motivos do baixo provimento e fixação de profissionais de saúde nas áreas mais remotas do Amazonas, uma vez que estes preferem capitais e centros urbanos mais desenvolvidos e com maiores investimentos na $A B$ e na capacitação e qualificação profissional, o que não é uma realidade nos interiores do Amazonas, porém, muito necessário considerando as populações especificas de indígenas, ribeirinhos e rurais que caracterizam essas regiões mais afastadas (Dolzane \& Schweickardt, 2020).

A organização da equipe multiprofissional é fragmentada pela intensa rotatividade e baixa fixação dos profissionais da saúde no Amazonas, que em sua grande maioria são remanescentes de outros estados, recém formados em busca de oportunidades de emprego, assim acontece também o desligamento de profissionais do seu posto sem ser substituído por outro profissional. Estas adversidades enfraquecem o vínculo profissional-paciente ou ESF-população essencial na AB (Dolzane \& Schweickardt, 2020).

A ESF conta com o protagonismo essencial do profissional Enfermeiro, uma vez que se refere ao desenvolvimento das atividades promotoras de saúde, onde é considerado o principal responsável pela realização de tais ações, além de ser visto como um educador por excelência a influenciar as maneiras como ações de promoção de saúde são pensadas e desenvolvidas, viabilizando a capacidade de autocuidado e melhora da qualidade de vida da população (Silva et al., 2020). Juntamente com os 
agentes comunitários de saúde (ACS) e o Núcleo de Ampliado de Saúde da família (NASF) desempenham um papel primordial nas fragilidades existentes a reabilitação e assistência adequada de acordo com a demanda, como por exemplo: a saúde da população idosa. (Franco et al., 2020). Sabendo das modificações em que o NASF passa a ser decisão da gestão municipal, alerta-se para o desafio da possível perda deste apoio as equipes de ESF.

O estudo recente de Ferreira (2021), apresenta diversos obstáculos e dificuldades enfrentados por uma equipe de ESF no Amazonas, este revela que as dificuldades com a estrutura física moldam os diferentes formatos das equipes e sua atuação. Tal fato é corroborado pela concordância de todos os profissionais de saúde que o meio ambiente e a questão ambiental são fundamentais para atingir os objetivos da ESF (Mendonça et al., 2012). Ainda no estudo Ferreira (2021), destaca dificuldades de investimento e materiais, realização de ações e dificuldades pessoais de criatividade e capacitação da equipe.

O investimento na formação de profissionais especializados para a qualificação do serviço na atenção primária de saúde é uma estratégia relevante pois, aumenta o grau de orientação ofertados aos usuários deste serviço, a ampliação da cobertura da ESF na unidade básica de (UBS) segue um maior grau de satisfação pois abrange uma cobertura menor de pacientes com a inclusão de ACS e profissionais generalistas que promovem uma cobertura com maior qualidade a saúde da população (Castro et al., 2014).

\section{Conclusão}

Partindo das características geográficas e socioeconômicas voltadas para as peculiaridades e modo de vida da população, a atenção primária no Amazonas enfrenta diversas adversidades referentes a um cenário limitado de recursos, transporte, equipamentos, acessibilidade e mobilidade, em meio a este paradigma encontrando, a equipe busca se tornar cada vez mais capaz em tomar decisões resolutivas, oportunas e necessárias para prestar assistência às populações residentes em áreas de difícil acesso. Tais fatores retratados exigem maiores investimentos, planejamentos, adaptações e capacitação dos profissionais pois a equipe multiprofissional trabalha em conjunto, fortalecendo vínculos com a comunidade, promovendo promoção, prevenção e qualidade da assistência em saúde, em especial em populações mais vulneráveis.

A dificuldade de acesso encontrada em famílias residentes nestas áreas remotas é um desafio a ser enfrentado pela gestão, visto que necessita de um planejamento estratégico eficaz que supra a maioria das necessidades da assistência em saúde. Portanto, é de extrema importância que os profissionais de saúde busquem articular as diretrizes políticas e as práticas de saúde, procurando ampliá-las através de pesquisas e criação de tecnologias que possibilitem a reorganização das práticas de saúde da família.

Através deste estudo e a escassez de trabalhos publicados referentes a situação da ESF no Amazonas, o estudo sobre atenção primaria no Amazonas abre um leque de interrogações para a busca de soluções adequada para abrangência adequada a saúde no Amazonas. Portanto, sugerem-se pesquisas e práticas voltadas para a temática, cujo intuito seja identificar os impasses encontrados pela população, além das dificuldades encontradas para o meio de trabalho da equipe e para uma busca de um conhecimento esclarecedor da temática e melhorias na implementação de programas e assistências qualificadas no atendimento à saúde pública da população.

\section{Referência}

Brasil (2017). Portaria no 2.436, de 21 de setembro de 2017. Aprova a Política Nacional de Atenção Básica, instituindo a revisão das diretrizes para a organização da Atenção Básica, dentro abrangência do Sistema Único de Saúde.

Brasil. (2011). Portaria $\mathrm{n}^{\circ}$ 2.488, de 21 de outubro de 2011. Aprova a Política Nacional de Atenção Básica, estabelecendo a revisão de diretrizes e normas para a organização da Atenção Básica, para a Estratégia Saúde da Família (ESF) e o Programa de Agentes Comunitários de Saúde (PACS). Diário Oficial da União, (204). 
Research, Society and Development, v. 10, n. 10, e333101017441, 2021

(CC BY 4.0) | ISSN 2525-3409 | DOI: http://dx.doi.org/10.33448/rsd-v10i10.17441

Castro, R. C. L. D., Knauth, D. R., Harzheim, E., Hauser, L., \& Duncan, B. B. (2012). Avaliação da qualidade da atenção primária pelos profissionais de saúde: comparação entre diferentes tipos de serviços. Cadernos de Saúde Pública, 28, 1772-1784.

Costa Franco, P., Esteves, A. V. F., de Oliveira, A. P. P., Sampaio, S. N., \& de Lima, E. S. (2020). Cotidiano do enfermeiro no atendimento ao idoso na estratégia saúde da família em manacapuru-amazonas. Cogitare Enfermagem, 25.

Dolzane, R. D. S. (2019). Provimento e fixação de profissionais de saúde na atenção básica em contextos de difícil acesso, no estado do Amazonas. e-gestor atenção básica espaço para informação e acesso aos sistemas da atenção básica., dezembro de 2020.

Estrela, C. (2018). Metodologia científica: Ciência, ensino, Pesquisa. Editora Artes Médicas.

Fausto, M. C. R., Giovanella, L., Mendonça, M. H. M. D., Seidl, H., \& Gagno, J. (2014). A posição da Estratégia Saúde da Família na rede de atenção à saúde na perspectiva das equipes e usuários participantes do PMAQ-AB. Saúde em debate, 38, 13-33.

Ferreira, D. S., Ramos, F. R. S., Teixeira, E., Monteiro, W. F., \& de Aguiar, A. P. (2021). Obstáculos para práxis educativa de enfermeiros na Estratégia Saúde da Família. Revista Gaúcha de Enfermagem, 42.

Figueira, M. C. E. S., Marques, D., Vilela, M. F. G., Pereira, J. D. A., Bazílio, J., \& Silva, E. M. (2020). Fluvial family health: work process of teams in riverside communities of the Brazilian Amazon. Rural and Remote Health, 20(3), 5522-5522.

Garnelo, L., Lucas, A. C. D. S., Parente, R. C. P., Rocha, E. S. C., \& Gonçalves, M. J. F. (2014). Organização do cuidado às condições crônicas por equipes de Saúde da Família na Amazônia. Saúde em Debate, 38, 158-172.

Guimarães, W. S. G. (2016). Avaliação da Atenção Pré-natal na Atenção Básica por macroregiões geográficas e por regiões de saúde (Doctoral dissertation).

Instituto Brasileiro De Geografia E Estatística (IBGE). (2010) Amazonas, 2010.

Macinko, J., \& Harris, M. J. (2015). Brazil's family health strategy-delivering community-based primary care in a universal health system. N Engl J Med, $372(23), 2177-81$.

Mendonça, R. D. C., Giatti, L. L., \& Toledo, R. F. D. (2012). A temática ambiental em representações e práticas de profissionais de saúde da família no município de Manaus-AM/Brasil. Saúde e Sociedade, 21, 776-787.

Moretti-Pires, R. O., \& Campos, D. A. D. (2010). Equipe multiprofissional em Saúde da Família: do documental ao empírico no interior da Amazônia. Revista brasileira de educação médica, 34(3), 379-389.

Oliveira, H. M. D., Gonçalves, M. J. F., \& Pires, R. O. M. (2011). Caracterização da estratégia saúde da família no estado do Amazonas, Brasil: análise da implantação e impacto. Cadernos de Saúde Pública, 27(1), 35-45.

Rabelo, A. L. R., Lacerda, R. A., Rocha, E. S. C., Gagno, J., Fausto, M. C. R., \& Gonçalves, M. J. F. (2020). Care coordination and longitudinality in primary health care in the Brazilian Amazon. Revista brasileira de enfermagem, 73(3).

Silva, C. R., Carvalho, B. G., Cordoni Júnior, L., \& Nunes, E. D. F. P. D. A. (2017). Dificuldade de acesso a serviços de média complexidade em municípios de pequeno porte: um estudo de caso. Ciência \& Saúde Coletiva, 22, 1109-1120.

Silva, N. C. D. C. D., Mekaro, K. S., Santos, R. I. D. O., \& Uehara, S. C. D. S. A. (2020). Knowledge and health promotion practice of Family Health Strategy nurses. Revista Brasileira de Enfermagem, 73(5).

Tonhá, A. C. M., de Souza Júnior, P. R. B., Bezerra, A. L. Q., de Pinho Santos, T. A., \& Rosso, C. F. W. (2015). Acesso aos serviços de saúde nos municípios do entorno sul do Distrito Federal. Revista Eletrônica de Enfermagem, 17(2), 238-46. 